\title{
Expected performance of the AugerPrime Radio Detector
}

\author{
Felix Schlüter ${ }^{a, b, *}$ on behalf of the Pierre Auger Collaboration \\ (a complete list of authors can be found at the end of the proceedings) \\ ${ }^{a}$ Karlsruhe Institute of Technology, Institute for Astroparticle Physics, Karlsruhe, Germany \\ ${ }^{b}$ Universidad Nacional de San Martín, Instituto de Tecnologías en Detección y Astropartículas, \\ Buenos Aires, Argentina \\ Observatorio Pierre Auger, Av. San Martín Norte 304, 5613 Malargüe, Argentina \\ E-mail: spokespersons@auger.org
}

The AugerPrime Radio Detector will significantly increase the sky coverage of mass-sensitive measurements of ultra-high energy cosmic rays with the Pierre Auger Observatory. The detection of highly inclined air showers with the world's largest $3000 \mathrm{~km}^{2}$ radio-antenna array in coincidence with the Auger water-Cherenkov detector provides a clean separation of the electromagnetic and muonic shower components. The combination of these highly complementary measurements yields a strong sensitivity to the mass composition of cosmic rays. We will present the first results of an end-to-end simulation study of the performance of the AugerPrime Radio Detector. The study features a complete description of the AugerPrime radio antennas and reconstruction of the properties of inclined air showers, in particular the electromagnetic energy. The performance is evaluated utilizing a comprehensive set of simulated air showers together with recorded background. The estimation of an energy- and direction-dependent aperture yields an estimation of the expected 10-year event statistics. The potential to measure the number of muons in air showers with the achieved statistics is outlined. Based on the achieved energy resolution, the potential to discriminate between different cosmic-ray primaries is presented.

$37^{\text {th }}$ International Cosmic Ray Conference (ICRC 2021)

July 12th - 23rd, 2021

Online - Berlin, Germany

\footnotetext{
${ }^{*}$ Presenter
} 


\section{Introduction}

The AugerPime Radio Detector (RD) will explore radio detection of Extensive Air Showers (EAS) on a very large scale with 1661 radio antennas covering an area of $3000 \mathrm{~km}^{2}$ [1], thus providing sufficient aperture to collect sizable statistics of cosmic rays at the highest energies beyond $10^{19} \mathrm{eV}$. The deployment of the radio antennas will extend the sky coverage of masssensitive measurements with the Pierre Auger Observatory towards the horizon $\left(\theta \sim 65^{\circ}-85^{\circ}\right)$, an advantageous capacity for anisotropy studies.

The detection of highly inclined EAS with radio antennas allows a clean measurement of the electromagnetic shower component. The radio emission, which propagates (almost) without attenuation through the atmosphere, illuminates large areas. These properties allow for an accurate determination of the cosmic-ray energy with a small dependency on the particle mass and with a small theoretical uncertainty. In contrast, the Auger Surface Detector (SD) is sensitive to the muonic shower component reaching the ground while the electromagnetic cascade is absorbed in the atmosphere. The combination of the RD and SD provides a very pure electron-muon separation which offers a wealth of information, in particular about the mass of the primary cosmic ray.

In this work, we investigate the expected performance of the AugerPrime Radio Detector on simulated events and outline the potential of hybrid detections with the RD and SD.

\section{Simulation study of the AugerPrime Radio Detector}

The AugerPrime Radio Detector will consist of 1661 dual-polarized Short Aperiodic Loaded Loop Antennas (SALLAs) mounted on top of the SD stations. The antennas are fully integrated with the SD stations and will receive triggers from the water-Cherenkov detectors (WCDs). Currently, four SALLAs are in operation in the $1500 \mathrm{~m}$-grid, this entails the recording of the typical radio background at the site, including ambient narrow- and broad-band radio-frequency interference (RFI). A description of the technical design of the RD and calibration (used in this study), the operation of the engineering array, and a first analysis of its data can be found in reference [2].

The operation of the RD and the analysis of its data builds on the longstanding expertise acquired with the Auger Engineering Radio Array (AERA). For the following study, the Auger reconstruction and simulation framework $\overline{\mathrm{Off}}$ line [3], which has been extended to also serve the needs of the RD, is utilized.

Complete end-to-end simulation study: First, the particle and radio-emission footprints of an EAS are simulated for the location and detector layout of the Pierre Auger Observatory (cf. next par.). This is followed by a detector simulation and event reconstruction with the SD $[4,5]$. If successful, a detector simulation of the RD is conducted for all stations for which the WCD is triggered. Details about the simulation of radio antennas with $\overline{\mathrm{Off}} \underline{\text { line }}$ can be found in reference [3]. The signal are smeared in each antenna polarisation independently by multiplying the uncalibrated signal traces with a normally-distributed factor $\mathcal{N}\left(1, \sigma_{A}=0.05\right)^{1}$ per antenna. The station timing is smeared by adding a normally-distributed time jitter of $\mathcal{N}\left(0, \sigma_{t}=6\right) \mathrm{ns}$. This procedure adequately describes antenna-to-antenna variation in the detector description. An antenna-to-antenna variation in the amplitude of $\sigma_{A}=5 \%$ was found for the AERA-Butterfly antennas [6, Fig. 4.8], however it is still under investigation whether this value is sufficient to describe the variation in the overall

\footnotetext{
${ }^{1}$ This spread of $\sigma_{A}=5 \%$ corresponds to a spread of $\sigma_{f}=10 \%$ on the energy fluence.
} 

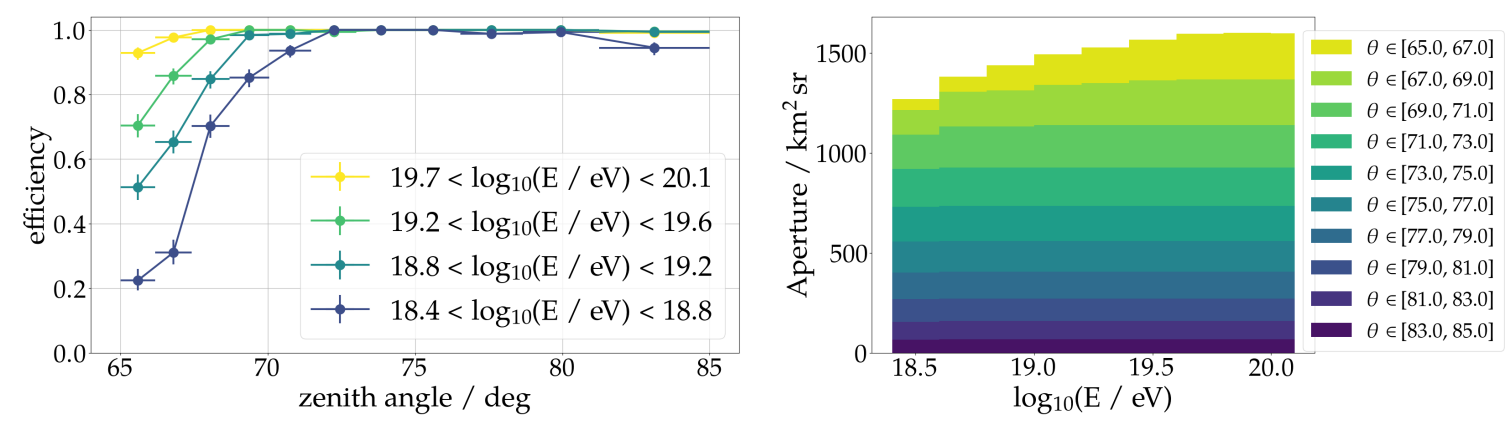

Figure 1: Left: Detection efficiency for the RD as a function of the zenith angle for different energy bins. Right: Stacked histogram of the aperture. Each layer shows the contribution of the corresponding zenith angle bin to the overall aperture as a function of the energy. For illustration purposes, in both figures, alternative binning (in energy and zenith angle) is used to calculate the event statistics (cf. Fig. 2).

antenna responses of the RD stations, including effects of a changing directional sensitivity due to mechanical distortion, mechanical differences between the upgraded SD stations, and/or changing reflection properties of the surrounding ground. The detector simulation includes the addition of in-situ measured ambient background to the simulated radio signals ${ }^{2}$. Given the raw signal traces, the electric field traces are reconstructed using the sensitivity pattern of the antenna and the SDreconstructed shower direction. From these calibrated signal traces, a set of quantities such as the energy fluence $f / \mathrm{eV} \mathrm{m}^{-2}$, i.e., the energy deposit of the radiation energy in the frequency band of 30 to $80 \mathrm{MHz}$ per unit area, is reconstructed for each detector station. No signal cleaning or RFI suppression procedures are applied. Employing a sophisticated cleaning algorithm will thus likely improve the reconstruction in the future, especially at lower energies. In the following, we estimate the detection efficiency and achievable event statistics (cf. Sec. 3) from the reconstructed signals and conduct a full event reconstruction (cf. Sec. 4).

The set of inclined air shower simulations is generated with CORSIKA/CoREAS v7.7401 [7]. The ambient conditions and the detector layout are chosen as relevant for the Pierre Auger Observatory. The showers are initiated by four primaries: proton, helium, nitrogen, and iron and two different high energy hadronic interaction models, QGSJETII-04 and Sibyll 2.3d, together with the low energy model UrQMD are used. An optimized thinning level of $10^{-6}$ is set. For each of these combinations, we simulated $\sim 2000$ showers with a total of 15969 showers. The showers cover the energy range between $10^{18.4} \mathrm{eV}$ and $10^{20.1} \mathrm{eV}$, uniformly randomized in $\log _{10}(E / \mathrm{eV})$. The arrival directions are isotropic between zenith angles of $65^{\circ}$ and $85^{\circ}$. The shower impact point at the ground, the "core", is randomly distributed within the finite $3000 \mathrm{~km}^{2} \mathrm{SD}$ array. For each shower, all antennas are simulated within a geometry-dependent maximum distance to the shower axis. In the following study, unless explicitly stated otherwise, only the 7972 QGSJET showers are used.

\section{Detection of inclined air showers with the AugerPrime Radio Detector}

To detect a cosmic-ray induced air shower with the $\mathrm{RD}$, we require three "signal" stations with a radio signal surpassing a signal-to-noise $\mathrm{SNR}=\left(A_{\max }^{\mathrm{sig}} / A_{\mathrm{RMS}}^{\mathrm{noise}}\right)^{2}>10$ with $A_{\max }^{\text {sig }}$ being the maximum of the Hilbert envelope of the horizontal electric field traces within a pre-defined signal

\footnotetext{
${ }^{2}$ In total 2738 traces of $8.2 \mu$ s each per polarisation from one RD station are used for $\sim 85000$ simulated signals.
} 


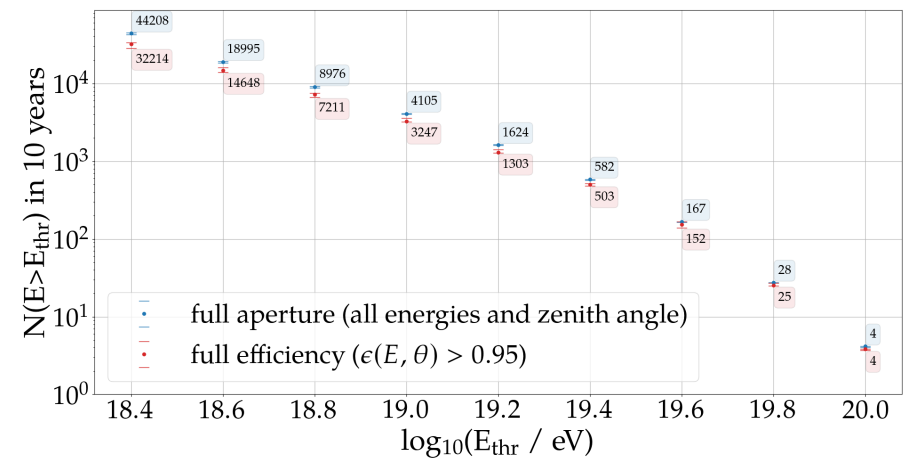

Figure 2: Integrated 10-year event statistics as a function of the lower threshold energy. We calculate the event statistics for the full aperture (blue), i.e., for all available energies and zenith angles, and again only for all zenith angle and energy bins in which the estimated efficiency is above $95 \%$ (red).

window, and $A_{\mathrm{RMS}}^{\text {noise }}$ being the root-mean-square amplitude of the same traces in a time interval detached from the signal region, i.e., a noise window. In addition to that, the underlying WCD had to trigger and the SD event reconstruction had to be successful.

The detection efficiency is shown in Fig. 1 (left) as a function of the zenith angle ( $\mathrm{x}$-axis, binned in $\sin ^{2} \theta$ ) and energy (color-coded) for all events reconstructed by the SD. This aspect may overestimate the true RD efficiency. However, due to the known muon deficit in the simulations [8], the SD efficiency, and thereby the RD efficiency is underestimated. By normalizing the RD efficiency to all SD-reconstructed showers, we mitigate this effect. From measurements, we know that the SD is fully efficient beyond an energy of $10^{18.6} \mathrm{eV}$ [5]. The uncertainties are calculated assuming binominal statistics.

The aperture is calculated based on the estimated efficiency $\epsilon\left(\theta_{1}, \theta_{2}, E_{1}, E_{2}\right)$ according to Eq. (1),

$$
\mathcal{A}\left(\theta_{1}, \theta_{2}, E_{1}, E_{2}\right)=3000 \mathrm{~km}^{2} \pi\left[\cos ^{2} \theta_{1}-\cos ^{2} \theta_{2}\right] \epsilon\left(\theta_{1}, \theta_{2}, E_{1}, E_{2}\right) .
$$

This equation accounts for the fact that with an increasing zenith angle the array projection into the shower frame becomes smaller $(\sim \cos \theta)$. Hence the aperture is, as we consider only "contained" events, limited at higher zenith angles ${ }^{3}$. Fig. 1 (right) shows the aperture $\mathcal{A}\left(\theta_{1}, \theta_{2}, E_{1}, E_{2}\right)$ as a function of energy in a stacked histogram in which each (colored) layer shows the contribution of the corresponding zenith angle bin to the overall aperture. If the layer is flat, full efficiency over the full energy range for this zenith angle range is achieved. A maximum of around $1600 \mathrm{~km}^{2} \mathrm{sr}$ is reached. The 10-year event statistics are calculated with the estimated aperture and the Auger-measured flux of ultra-high energy cosmic rays [9]. The integrated event number above a given energy $E_{\text {thr }}$ is shown in Fig. 2. We find that the RD will detect $4105_{-89}^{+45}\left(8976_{-224}^{+131}\right)$ events for energies above $10^{19} \mathrm{eV}\left(10^{18.8} \mathrm{eV}\right)$ in a 10-year life span with full aperture. The uncertainties are obtained by systematically shifting the efficiency by its 1-sigma statistical uncertainty and re-evaluating the event statistics. No Poisson uncertainty, as appropriate when measuring the event statistics, is folded in. These results are in good agreement with the study published in Ref. [1] which estimated the achievable event statistics with an analytical model of the radio emission and without conducting a detector simulation or adding ambient background noise. With the Sibyll-2.3d showers, we obtain comparable numbers: $4047_{-93}^{+49}\left(8839_{-220}^{+130}\right)$. 

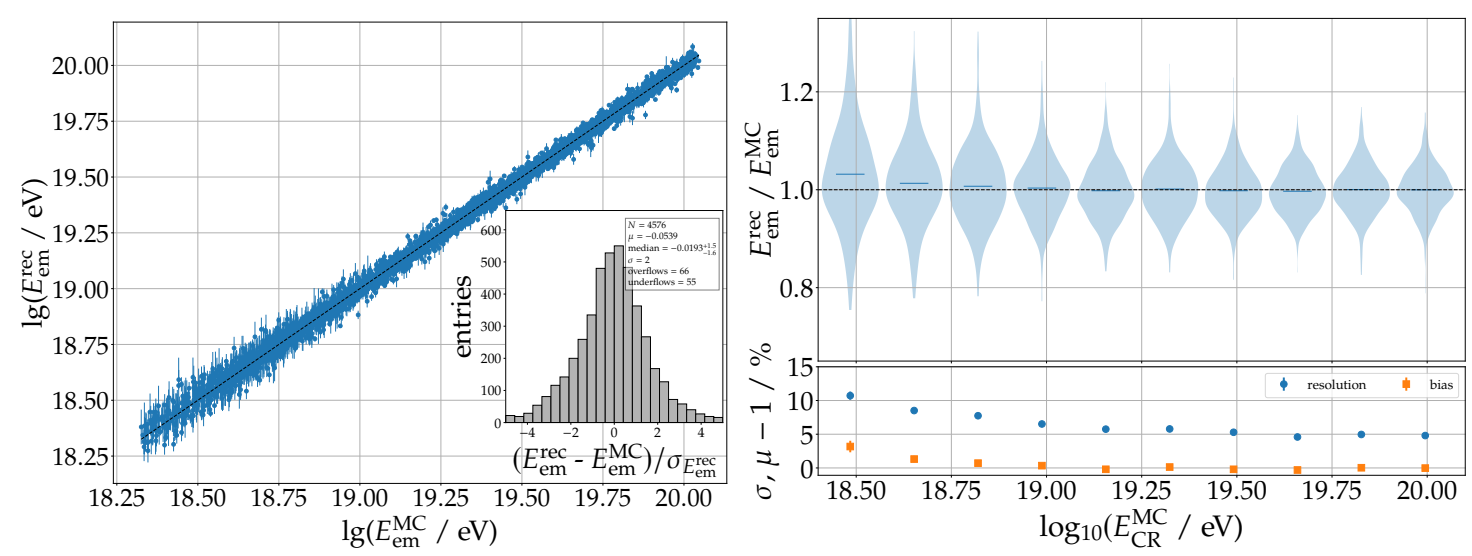

Figure 3: Left: Scatter of the radio-reconstructed electromagnetic energy vs the true electromagnetic energy (dashed black line indicates the diagonal). Right: Violin histograms of the energy reconstruction as a function of the true cosmic-ray energy (top). The reconstruction bias and resolution are shown in the bottom panel.

\section{Event reconstruction of inclined air showers}

The arrival direction is fitted with a model which assumes a spherical expansion from a pointlike source. Initial values for the fit are taken from the reconstructed SD shower axis. We found good agreement between the radio-reconstructed and true arrival direction with a resolution of $\sigma_{68 \%}\left(\measuredangle\left(\hat{a}_{\mathrm{RD}}, \hat{a}_{\mathrm{MC}}\right)\right)=0.19^{\circ}$ for all showers with $\theta_{\mathrm{RD}} \geq 68^{\circ}$ and at least 5 signal stations. To reconstruct the shower energy, we fit a lateral distribution function (LDF) which describes the asymmetric signal distribution of the radio emission in the $\vec{v} \times \vec{B}$-polarisation ${ }^{4}$ by an explicit decomposition of symmetric and asymmetric components and an individual description of them. For a given geometry (core and axis), the LDF describes the entire footprint with two fit parameters: the geomagnetic radiation energy $E_{\text {geo }}$, and the geometrical distance between the core and the shower maximum $d_{\max }$. More information regarding the LDF model can be found in Ref. [10]. In practice, the core is also fitted (starting from the SD-reconstructed core) with fixed RD-reconstructed arrival direction amounting to a total of 4 fit parameters. Initial values for $E_{\text {geo }}$ and $d_{\max }$ are derived using the reconstructed SD energy and RD arrival direction. Furthermore, the $\chi^{2}$ minimisation features several steps in which the core and/or $d_{\max }$ are fixed to improve the robustness on the fit. For the reconstruction, the simulated atmospheric model and magnetic field are known. However, both can be modelled with high accuracy and will not significantly affect the reconstruction when relying on their models. Furthermore, we add the lowest signal $f_{\vec{v} \times \vec{B}}(\mathrm{SNR}>10)$ to the uncertainty of each signal to mitigate the effect of thinning artifacts [10].

The geomagnetic radiation energy $E_{\text {geo }}$ can be used to reconstruct the electromagnetic shower energy $E_{\mathrm{em}}$ via an approximately quadratic relationship $E_{\text {geo }}^{2} \sim E_{\mathrm{em}}$. However, $E_{\text {geo }}$ also has a second-order dependency on the magnetic field (strength and orientation) and the atmospheric density $\rho_{\max }$ at which the bulk of the radio signals are emitted ( $\approx$ shower maximum) [11]. Therefore, we define the corrected geomagnetic radiation energy $S_{\text {geo }}^{\rho}$ which is then calibrated to the (simulated)

\footnotetext{
${ }^{3}$ If this condition can be lifted in the future, i.e., considering also showers for which only a part of the footprint overlaps with the array, the aperture will significantly increase at higher zenith angles.

${ }^{4}$ With $\vec{v}$ being the direction of the shower particles and $\vec{B}$ the orientation of the magnetic field vector.
} 
electromagnetic energy with a power law (cf. Eq. (2)),

$$
S_{\text {geo }}^{\rho}=\frac{E_{\text {geo }}\left(\sin ^{2} \alpha\left(\frac{|\vec{B}|}{23.759 \mathrm{mG}}\right)^{1.8}\right)^{-1}}{\left(1+p_{0}\left(\exp \left[p_{1}\left(\rho_{\max }-\langle\rho\rangle\right]-1\right)\right)^{2}\right.}, \quad E_{\mathrm{em}}=\left(S_{\text {geo }}^{\rho} / S_{19}\right)^{(1 / \gamma)} 10 \mathrm{EeV} .
$$

The angle between the shower axis and the magnetic field vector is donated by $\alpha$. For a given atmospheric model and shower axis, $\rho_{\max }$ is determined from the fitted $d_{\max }$. The parameters $S_{19}$, $\gamma, p_{0}$, and $p_{1}$ can be found with a combined fit of the reconstructed $E_{\mathrm{geo}}, \rho_{\max }$, and the simulated $E_{\text {em }}$ while fixing $\left\langle\rho_{\text {max }}\right\rangle=0.3 \mathrm{~kg} \mathrm{~m}^{-3}$. However, we found that with the reconstructed $E_{\text {geo }}, \alpha$, and $\rho_{\max }$, we do not achieve an unbiased reconstruction of $E_{\mathrm{em}}$ when fitting the 4 parameters all together. Therefore, we fit only $S_{19}=3.095 \pm 0.005 \mathrm{GeV}, \gamma=2.0046 \pm 0.0010$ and fix $p_{0}=0.497$ and $p_{1}=-2.737 /\left(\mathrm{kg} \mathrm{m}^{-3}\right)^{5}$. Before evaluating the reconstruction accuracy, a selection is applied with the main criteria being: $n_{\text {stat }}^{\mathrm{RD}}(\mathrm{SNR}>10) \geq 5, \theta_{\mathrm{RD}} \geq 68.0^{\circ}, \alpha_{\mathrm{RD}}>20.0^{\circ}$, $\measuredangle\left(\hat{a}_{\mathrm{RD}}, \hat{a}_{\mathrm{SD}}\right)<1.50^{\circ}{ }^{6}$. The total selection has an efficiency of $57.4 \%$ (of this $69.2 \%$ are cut by $\left.n_{\text {stat }}^{\mathrm{RD}}(\mathrm{SNR}>10) \geq 5\right)^{7}$. Fig. 3 (left) shows the reconstructed electromagnetic energy as a function of the simulated electromagnetic energy demonstrating a good correlation. The inset shows the pull-distribution with a standard deviation of $\sigma=2$ indicating that the uncertainties are currently underestimated. They are calculated using the statistical uncertainties of $E_{\text {geo }}$ and $\rho_{\text {max }}$ derived from the $\chi^{2}$ minimisation and propagated to $E_{\mathrm{em}}$ using Gaussian error propagation and Eq. (2). This may also affect the statistical uncertainties of the fit parameter $S_{19}, \gamma$ which seem underestimated. The resolution and bias, together with the distribution of $E_{\mathrm{em}} / E_{\mathrm{em}}^{\mathrm{MC}}$ binned in $\log _{10}\left(E_{\mathrm{CR}}^{\mathrm{MC}}\right)$ is shown in Fig. 3 (right). The resolution improves with energy to below 10\%, as expected as the impact of the ambient background decreases. Furthermore, we do not find any bias in the reconstruction for the different primaries and no significant variation in resolution.

Finally, the cosmic-ray energy is estimated from the electromagnetic energy with $E_{\mathrm{CR}}=$ $E_{\mathrm{em}}\left(1.1426-0.0327 \log _{10}\left(E_{\mathrm{em}} / 10 \mathrm{EeV}\right)\right)$, which is derived from simulations. We note that this function is not universal for all primaries and that it introduces a mass-dependent bias to the reconstructed cosmic-ray energy of below $\pm 3 \%$ and hence a slight worsening of the resolution.

We found no difference in the reconstruction of the electromagnetic energy for the showers simulated with Sibyll-2.3d. Applying an amplitude smearing of $\sigma_{A}=10 \%$ instead of $\sigma_{A}=5 \%$ as part of the detector simulation (cf. Sec. 2) has a mild effect on the overall energy resolution which degrades from $6 \%$ (for $\sigma_{A}=5 \%$ ) to $9 \%$.

\section{Hybrid measurements of inclined air showers with AugerPrime}

To demonstrate the potential of RD-SD hybrid measurements, we mimic the energy spectrum the RD will measure in the future by a energy-dependent reweighting of our simulations with the estimated 10-years event statistics. This procedure necessitates the use of the same reconstructed shower several times at lower energies and introduces a randomness to the results.

\footnotetext{
${ }^{5}$ The values for $p_{0}$ and $p_{1}$ are obtained from a combined fit to data reconstructed without any detector simulation, for which no noise was added to the simulated radio signals, and the true arrival direction was known, Ref. [10]

${ }^{6}$ In addition, we ensure an sufficient sampling of the LDF around the shower axis.

${ }^{7}$ We note that this selection is not completely equally efficient for all primaries with a variation on the \%-level. An unequal efficiency poses problems when studying the cosmic-ray composition (cf. Sec. 5) and has to be addressed in the future.
} 


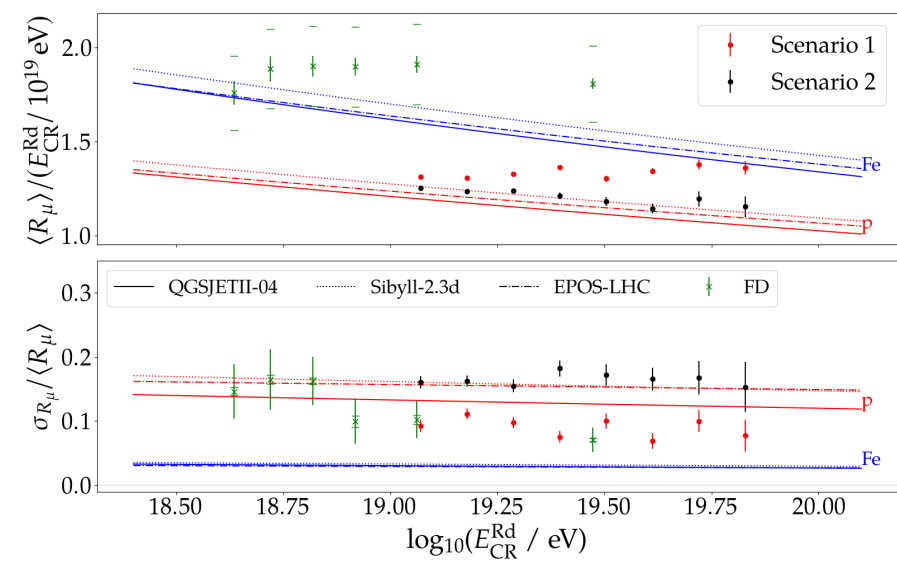

Figure 4: Simulated measurement of the relative mean number of muons with the SD and RD for two different composition scenarios comprised of $\mathrm{p}, \mathrm{He}, \mathrm{N}$, and Fe primaries shown alongside the most recent measurements by the FD and SD [12] and theoretical predictions for pure proton and iron compositions. The error bars illustrate the statistical uncertainty only and the errorcaps (only shown for SD-FD, green) show the systematic uncertainty.

The relative number of muons $\left\langle R_{\mu}\right\rangle /\left(E_{\mathrm{CR}} / 10 \mathrm{EeV}\right)$ and their (physical) fluctuations $\sigma_{R_{\mu}} /\left\langle R_{\mu}\right\rangle$ in inclined air showers are composition-sensitive observables. Measurements of these observables with the Auger Fluorescence Detector (FD) and the SD have been shown to disagree with the theoretical predictions and hint at a heavier composition at the highest energies [8, 12]. However, the present data of FD-SD lacks statistics at the highest energies due to the lower exposure of the FD. Thus, data with RD-SD will increase the available statistics by more than one order of magnitude at energies above $10^{19} \mathrm{eV}$. Here we adopt a simplified analysis strategy ${ }^{8}$ : The mean $\mu(x)$ and variance $V(x)$ of the relative muon number is obtained from $x \equiv R_{\mu} /\left(E_{\mathrm{CR}} / 10 \mathrm{EeV}\right)$ in bins of $\log _{10}\left(E_{\mathrm{CR}} / \mathrm{eV}\right)$. The physical fluctuations $\sigma$ are estimated by subtracting the detector $s_{R_{\mu}}$ and energy resolution $s_{E}$ from the variance $\sigma^{2} /\left\langle R_{\mu}\right\rangle^{2} \approx V(x) / \mu(x)^{2}-s_{R_{\mu}}^{2}-s_{E}^{2}$. Fig. 4 shows an example RD-SD measurement for two different astrophysical reference scenarios with different, energy dependent, abundances for $\mathrm{p}, \mathrm{He}, \mathrm{N}$, and $\mathrm{Fe}$, the so-called maximum-rigidity (1) and photodisintegration (2) scenarios [13, Fig. 2.10]. The green markers show the measurement with the FD-SD. No systematic uncertainty is shown for the RD-SD measurements but it is expected that they are at the same level as for the FD-SD (indicated by the errorcaps). It can be seen that with the statistics expected for the RD, this analysis can be extended to higher energies. Discrimination between these scenarios is especially possible using the physical fluctuations, which are much less affected by the systematic uncertainty in the energy scale.

The isolation of protons at the highest energies $\left(>10^{19} \mathrm{eV}\right)$ would, if they exist, enable cosmic-ray astronomy as these particles are less deflected by Galactic magnetic fields and thus indicate source directions. Protons, on average, produce fewer muons for a given energy than any other charged hadron. Thus $r\left(R_{\mu}, E_{\mathrm{em}}\right)=R_{\mu} / E_{\mathrm{em}}^{0.9}$ can be used to discriminate protons from other primaries ${ }^{9}$. In Fig. 5 (left), $r\left(R_{\mu}, E_{\mathrm{em}}\right)$ is shown as a function of $E_{\mathrm{em}}$ for a (randomly drawn) set of simulations of 50\% / 50\% proton and iron, following the expected RD energy spectrum. The transparency of the marker signifies how often a particular shower was drawn. The histograms on the y-axis show the projection of all showers onto this axis. The figure of merit (FOM) [13, Eq. (3.1)] for the separation of these two distributions with $r\left(R_{\mu}, E_{\mathrm{em}}\right)$ is $\mathrm{FOM}=1.48$. For 1000 random instances

\footnotetext{
${ }^{8}$ This ansatz is only strictly correct when $R_{\mu}$ scales linearly with $E_{\mathrm{CR}}$. For data this assumption is accurate, for simulations, not.

${ }^{9}$ The exponent of 0.9 is necessary because the $R_{\mu}$ does not scale linearly with $E_{\mathrm{em}}$ (in simulations) and will slightly depend on the hadronic interaction model.
} 

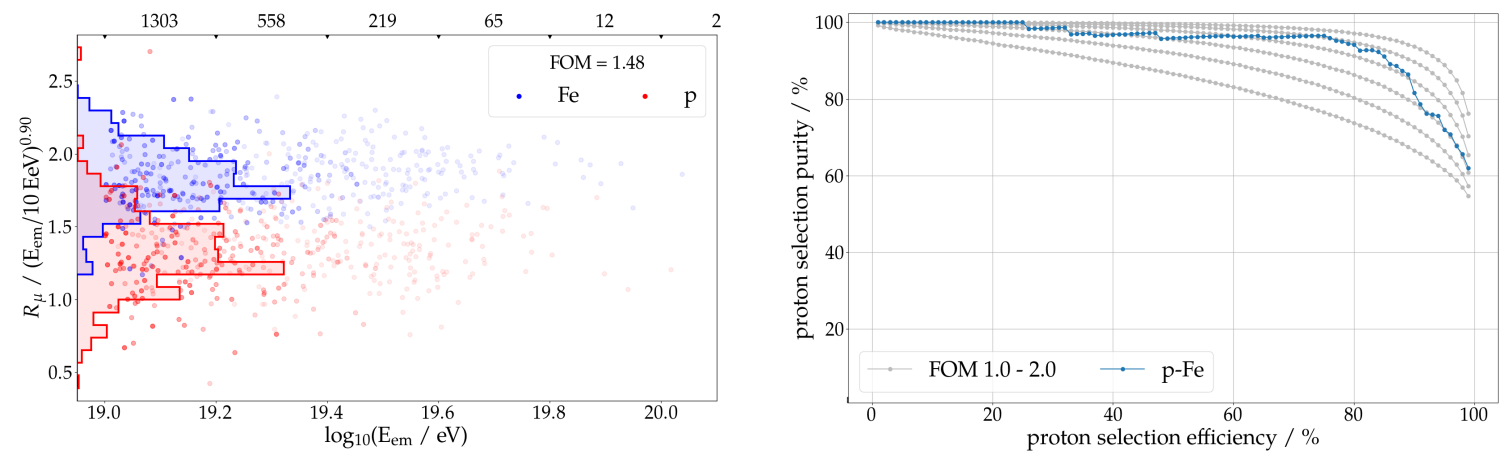

Figure 5: Left: Proton-iron separation via $r\left(R_{\mu}, E_{\mathrm{em}}\right)$ as function of $E_{\mathrm{em}}$. The transparency of the markers reflects their weight. Event number are shown at the top. The histograms indicate the energy independent separation of the two distributions. Right: Proton selection purity for $r\left(R_{\mu}, E_{\mathrm{em}}\right)$ as function of the efficiency. The grey lines show the expectation for toy data with a FOM of 1 to 2 .

of the dataset, we find $\langle\mathrm{FOM}\rangle \pm \sigma=1.51 \pm 0.05$. A FOM of 1.5 corresponds approximately to a separation of proton and iron with the shower maximum $X_{\max }$ measured with a detector resolution of $15 \mathrm{~g} \mathrm{~cm}^{-2}$. In Fig. 5 (right), the proton selection purity for $r\left(R_{\mu}, E_{\mathrm{em}}\right)$ as a function of the selection efficiency is shown, a $\sim 90 \%$ proton purity with a efficiency of $80 \%$ is achieved.

\section{Summary and conclusions}

We presented a first Monte-Carlo driven end-to-end simulation study of the AugerPrime Radio Detector. The use of CoREAS showers, a complete (radio) detector simulation, the addition of measured noise, and the complete radio-reconstruction of the air shower observables constitute a major improvement with respect to previous studies. The expected event statistics for the RD are in good agreement with previous studies and will enable an accurate measurement of the number of muons in inclined air showers in conjunction with the Auger Surface Detector, allowing a discrimination between different astrophysical scenarios. The first results of a full event reconstruction with a newly developed LDF model with the RD predict an energy resolution $\lesssim 10 \%$, given current assumptions. This resolution allows for a separation of proton and iron primaries with a proton purity of $\sim 90 \%$ at an efficiency of $80 \%$.

\section{References}

[1] Bjarni Pont [Pierre Auger Coll.], PoS (ICRC2019) 395.

[2] Tomáš Fodran [Pierre Auger Coll.], PoS (ICRC2021) 270.

[3] P. Abreu et al. [Pierre Auger Coll.], Nucl. Instrum. Meth. A 635 (2011) 92-102.

[4] S. Argirò et al., Nucl. Instrum. Meth. A 80 (2007) 1485-1496.

[5] A. Aab et al. [Pierre Auger Coll.], J. Cosmol. Astropart. P. 08 (2014) 019.

[6] F. Canfora, PhD Thesis, 2021 - NIKEF.

[7] T. Huege, M. Ludwig and C.W. James, AIP Conf. Proc. 1535 (2012) 128.

[8] A. Aab et al. [Pierre Auger Coll.], Phys. Rev. D 91 (2015) 059901.

[9] A. Aab et al. [Pierre Auger Coll.], Phys. Rev. D 102 (2020) 062005.

[10] T. Huege and F. Schlüter, PoS (ICRC2021) 209.

[11] Christian Glaser et al., J. Cosmol. Astropart. P. 09 (2016) 024.

[12] A. Aab et al. [Pierre Auger Coll.], Phys. Rev. Lett. 126 (2021) 152002.

[13] A. Aab et al. [Pierre Auger Coll.], arXiv:1604.03637. 


\section{The Pierre Auger Collaboration}

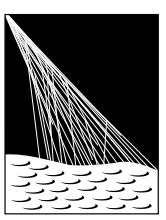

PIERRE

AUSGERVATORY

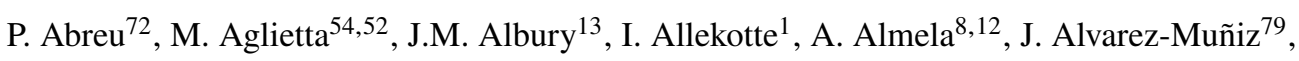
R. Alves Batista ${ }^{80}$, G.A. Anastasi ${ }^{63,52}$, L. Anchordoqui ${ }^{87}$, B. Andrada ${ }^{8}$, S. Andringa ${ }^{72}$, C. $\mathrm{Aramo}^{50}$, P.R. Araújo Ferreira ${ }^{42}$, J. C. Arteaga Velázquez ${ }^{67}$, H. Asorey $^{8}$, P. Assis ${ }^{72}$, G. Avila ${ }^{11}$, A.M. Badescu ${ }^{75}$, A. Bakalova ${ }^{32}$, A. Balaceanu ${ }^{73}$, F. Barbato ${ }^{45,46}$, R.J. Barreira Luz $^{72}$, K.H. Becker ${ }^{38}$, J.A. Bellido ${ }^{13,69}$, C. Berat ${ }^{36}$, M.E. Bertaina ${ }^{63,52}$, X. Bertou ${ }^{1}$, P.L. Biermann ${ }^{b}$, V. Binet ${ }^{6}$, K. Bismark ${ }^{39,8}$, T. Bister ${ }^{42}$, J. Biteau ${ }^{37}$, J. Blazek ${ }^{32}$, C. Bleve ${ }^{36}$, M. Boháčová ${ }^{32}$, D. Boncioli ${ }^{57,46}$, C. Bonifazi ${ }^{9,26}$, L. Bonneau Arbeletche ${ }^{21}$, N. Borodai ${ }^{70}$, A.M. Botti ${ }^{8}$, J. Brack ${ }^{d}$, T. Bretz ${ }^{42}$, P.G. Brichetto Orchera ${ }^{8}$, F.L. Briechle ${ }^{42}$, P. Buchholz ${ }^{44}$, A. Bueno ${ }^{78}$, S. Buitink ${ }^{15}$, M. Buscemi ${ }^{47}$, M. Büsken ${ }^{39,8}$, K.S. Caballero-Mora ${ }^{66}$, L. Caccianiga ${ }^{59,49}$, F. Canfora ${ }^{80,81}$, I. Caracas ${ }^{38}$, J.M. Carceller ${ }^{78}$, R. Caruso ${ }^{58,47}$, A. Castellina ${ }^{54,52}$, F. Catalanii ${ }^{19}$, G. Cataldi ${ }^{48}$, L. Cazon ${ }^{72}$, M. Cerda ${ }^{10}$, J.A. Chinellato ${ }^{22}$, J. Chudoba ${ }^{32}$, L. Chytka ${ }^{33}$, R.W. Clay ${ }^{13}$, A.C. Cobos Ceruttii ${ }^{7}$, R. Colalillo ${ }^{60,50}$, A. Coleman ${ }^{93}$, M.R. Coluccia ${ }^{48}$, R. Conceição ${ }^{72}$, A. Condorelli ${ }^{45,46}$, G. Consolati ${ }^{49,55}$, F. Contreras ${ }^{11}$, F. Convenga ${ }^{56,48}$, D. Correia dos Santos $^{28}$, C.E. Covault ${ }^{85}$, S. Dasso ${ }^{5,3}$, K. Daumiller ${ }^{41}$, B.R. Dawson ${ }^{13}$, J.A. Day ${ }^{13}$, R.M. de Almeida $^{28}$, J. de Jesús ${ }^{8,41}$, S.J. de Jong ${ }^{80,81}$, G. De Mauro ${ }^{80,81}$, J.R.T. de Mello Neto ${ }^{26,27}$, I. De Mitri ${ }^{45,46}$, J. de Oliveira ${ }^{18}$, D. de Oliveira Franco ${ }^{22}$, F. de Palma ${ }^{56,48}$, V. de Souza $^{20}$, E. De Vito ${ }^{56,48}$, M. del Río ${ }^{11}$, O. Deligny ${ }^{34}$, L. Deval ${ }^{41,8}$, A. di Matteo $^{52}$, C. Dobrigkeit ${ }^{22}$, J.C. D’Olivo ${ }^{68}$, L.M. Domingues Mendes ${ }^{72}$, R.C. dos Anjos ${ }^{25}$, D. dos Santos $^{28}$, M.T. Dova ${ }^{4}$, J. Ebr ${ }^{32}$, R. Engel ${ }^{39,41}$, I. Epicoco ${ }^{56,48}$, M. Erdmann ${ }^{42}$, C.O. Escobar ${ }^{a}$, A. Etchegoyen ${ }^{8,12}$, H. Falcke ${ }^{80,82,81}$, J. Farmer ${ }^{92}$, G. Farrar ${ }^{90}$, A.C. Fauth ${ }^{22}$, N. Fazzini ${ }^{a}$, F. Feldbusch ${ }^{40}$, F. Fenu ${ }^{54,52}$,

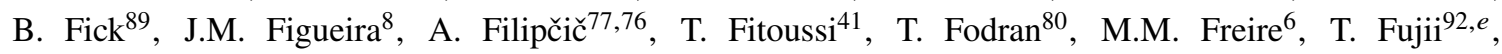
A. Fuster $^{8,12}$, C. Galea ${ }^{80}$, C. Galelli ${ }^{59,49}$, B. García ${ }^{7}$, A.L. Garcia Vegas ${ }^{42}$, H. Gemmeke ${ }^{40}$, F. Gesualdi ${ }^{8,41}$, A. Gherghel-Lascu ${ }^{73}$, P.L. Ghia ${ }^{34}$, U. Giaccari ${ }^{80}$, M. Giammarchi ${ }^{49}$, J. Glombitza ${ }^{42}$, F. Gobbi ${ }^{10}$, F. Gollan ${ }^{8}$, G. Golup ${ }^{1}$, M. Gómez Berisso ${ }^{1}$, P.F. Gómez Vitale ${ }^{11}$, J.P. Gongora ${ }^{11}$, J.M. González ${ }^{1}$, N. González ${ }^{14}$, I. Goos ${ }^{1,41}$, D. Góra ${ }^{70}$, A. Gorgi ${ }^{54,52}$, M. Gottowik ${ }^{38}$, T.D. Grubb ${ }^{13}$, F. Guarino ${ }^{60,50}$, G.P. Guedes ${ }^{23}$, E. Guido ${ }^{52,63}$, S. $\mathrm{Hahn}^{41,8}$, P. $\mathrm{Hamal}^{32}$, M.R. Hampel ${ }^{8}$, P. Hansen ${ }^{4}$, D. Harari ${ }^{1}$, V.M. Harvey ${ }^{13}$, A. Haungs ${ }^{41}$, T. Hebbeker ${ }^{42}$, D. Heck ${ }^{41}$, G.C. Hill ${ }^{13}$, C. Hojvat ${ }^{a}$, J.R. Hörandel ${ }^{80,81}$, P. Horvath ${ }^{33}$, M. Hrabovský ${ }^{33}$, T. Huege ${ }^{41,15}$, A. Insolia ${ }^{58,47}$, P.G. Isar $^{74}$, P. Janecek ${ }^{32}$, J.A. Johnsen ${ }^{86}$, J. Jurysek ${ }^{32}$, A. Kääpä ${ }^{38}$, K.H. Kampert ${ }^{38}$, N. Karastathis ${ }^{41}$, B. Keilhauer ${ }^{41}$, J. Kemp ${ }^{42}$, A. Khakurdikar ${ }^{80}$, V.V. Kizakke Covilakam $^{8,41}$, H.O. Klages ${ }^{41}$, M. Kleifges ${ }^{40}$, J. Kleinfeller ${ }^{10}$, M. Köpke ${ }^{39}$, N. Kunka ${ }^{40}$, B.L. Lago ${ }^{17}$, R.G. Lang ${ }^{20}$, N. Langner ${ }^{42}$, M.A. Leigui de Oliveira ${ }^{24}$, V. Lenok $^{41}$, A. Letessier-Selvon ${ }^{35}$, I. LhenryYvon $^{34}$, D. Lo Presti ${ }^{58,47}$, L. Lopes ${ }^{72}$, R. López ${ }^{64}$, L. Lu ${ }^{94}$, Q. Luce ${ }^{39}$, J.P. Lundquist ${ }^{76}$, A. Machado Payeras $^{22}$, G. Mancarella ${ }^{56,48}$, D. Mandat ${ }^{32}$, B.C. Manning ${ }^{13}$, J. Manshanden ${ }^{43}$, P. Mantsch ${ }^{a}$, S. Marafico ${ }^{34}$, A.G. Mariazzi ${ }^{4}$, I.C. Mariş ${ }^{14}$, G. Marsella ${ }^{61,47}$, D. Martello ${ }^{56,48}$, S. Martinelli ${ }^{41,8}$, O. Martínez Bravo ${ }^{64}$, M. Mastrodicasa ${ }^{57,46}$, H.J. Mathes ${ }^{41}$, J. Matthews ${ }^{88}$, G. Matthiae ${ }^{62,51}$, E. Mayotte ${ }^{38}$, P.O. Mazur ${ }^{a}$, G. MedinaTanco $^{68}$, D. Melo $^{8}$, A. Menshikov ${ }^{40}$, K.-D. Merenda ${ }^{86}$, S. Michal ${ }^{33}$, M.I. Micheletti ${ }^{6}$, L. Miramonti ${ }^{59,49}$, S. Mollerach ${ }^{1}$, F. Montanet ${ }^{36}$, C. Morello ${ }^{54,52}$, M. Mostafá ${ }^{91}$, A.L. Müller ${ }^{8}$, M.A. Muller ${ }^{22}$, K. Mulrey ${ }^{15}$, R. Mussa ${ }^{52}$, M. Muzio ${ }^{90}$, W.M. Namasaka ${ }^{38}$, A. Nasr-Esfahani ${ }^{38}$, L. Nellen ${ }^{68}$, M. Niculescu-Oglinzanu ${ }^{73}$, M. Niechciol ${ }^{44}$, D. Nitz ${ }^{89}$, D. Nosek ${ }^{31}$, V. Novotny ${ }^{31}$, L. Nožka ${ }^{33}$, A Nucita ${ }^{56,48}$, L.A. Núñez ${ }^{30}$, M. Palatka ${ }^{32}$, J. Pallotta ${ }^{2}$, P. Papenbreer ${ }^{38}$, G. Parente ${ }^{79}$, A. Parra ${ }^{64}$, J. Pawlowsky ${ }^{38}$, M. Pech ${ }^{32}$, F. Pedreira ${ }^{79}$, J. Pȩkala ${ }^{70}$, R. Pelayo ${ }^{65}$, J. Peña-Rodriguez ${ }^{30}$, E.E. Pereira Martins ${ }^{39,8}$, J. Perez Armand ${ }^{21}$, C. Pérez Bertolli $^{8,41}$, M. Perlin ${ }^{8,41}$, L. Perrone ${ }^{56,48}$, S. Petrera ${ }^{45,46}$, T. Pierog ${ }^{41}$, M. Pimenta ${ }^{72}$, V. Pirronello ${ }^{58,47}$, M. Platino ${ }^{8}$, B. Pont $^{80}$, M. Pothast ${ }^{81,80}$, P. Privitera ${ }^{92}$, M. Prouza ${ }^{32}$, A. Puyleart ${ }^{89}$, S. Querchfeld ${ }^{38}$, J. Rautenberg ${ }^{38}$, D. Ravignani ${ }^{8}$, M. Reininghaus ${ }^{41,8}$, J. Ridky ${ }^{32}$, F. Riehn ${ }^{72}$, M. Risse ${ }^{44}$, V. Rizi ${ }^{57,46}$, W. Rodrigues de Carvalho ${ }^{21}$, J. Rodriguez Rojo ${ }^{11}$, M.J. Roncoroni ${ }^{8}$, S. Rossoni ${ }^{43}$, M. Roth ${ }^{41}$, E. Roulet ${ }^{1}$, A.C. Rovero ${ }^{5}$, P. Ruehl ${ }^{44}$, A. Saftoiu ${ }^{73}$, F. Salamida ${ }^{57,46}$, H. Salazar ${ }^{64}$, G. Salina ${ }^{51}$, J.D. Sanabria Gomez ${ }^{30}$, F. Sánchez ${ }^{8}$, E.M. Santos ${ }^{21}$, E. Santos ${ }^{32}$, F. Sarazin ${ }^{86}$, R. Sarmento ${ }^{72}$, C. Sarmiento-Cano ${ }^{8}$, R. Sato ${ }^{11}$, 
P. Savina ${ }^{56,48,34,94}$, C.M. Schäfer ${ }^{41}$, V. Scherini ${ }^{56,48}$, H. Schieler ${ }^{41}$, M. Schimassek ${ }^{39,8}$, M. Schimp ${ }^{38}$, F. Schlüter ${ }^{41,8}$, D. Schmidt ${ }^{39}$, O. Scholten ${ }^{84,15}$, P. Schovánek ${ }^{32}$, F.G. Schröder ${ }^{93,41}$, S. Schröder ${ }^{38}$, J. Schulte ${ }^{42}$, S.J. Sciutto ${ }^{4}$, M. Scornavacche ${ }^{8,41}$, A. Segreto ${ }^{53,47}$, S. Sehgal ${ }^{38}$, R.C. Shellard ${ }^{16}$, G. Sigl ${ }^{43}$, G. Silli ${ }^{8,41}$, O. Sima $^{73, f}$, R. Šmída ${ }^{92}$, P. Sommers ${ }^{91}$, J.F. Soriano ${ }^{87}$, J. Souchard ${ }^{36}$, R. Squartini ${ }^{10}$, M. Stadelmaier ${ }^{41,8}$, D. Stanca ${ }^{73}$, S. Stanič ${ }^{76}$, J. Stasielak ${ }^{70}$, P. Stassi ${ }^{36}$, A. Streich ${ }^{39,8}$, M. Suárez-Durán ${ }^{14}$, T. Sudholz ${ }^{13}$, T. Suomijärvi ${ }^{37}$, A.D. Supanitsky ${ }^{8}$, Z. Szadkowski ${ }^{71}$, A. Tapia ${ }^{29}$, C. Taricco ${ }^{63,52}$, C. Timmermans ${ }^{81,80}$, O. Tkachenko ${ }^{41}$, P. Tobiska ${ }^{32}$, C.J. Todero Peixoto ${ }^{19}$, B. Tomé ${ }^{72}$, Z. Torrès ${ }^{36}$, A. Travaini ${ }^{10}$, P. Travnicek $^{32}$, C. Trimarelli ${ }^{57,46}$, M. Tueros ${ }^{4}$, R. Ulrich ${ }^{41}$, M. Unger ${ }^{41}$, L. Vaclavek ${ }^{33}$, M. Vacula ${ }^{33}$, J.F. Valdés Galicia ${ }^{68}$, L. Valore ${ }^{60,50}$, E. Varela ${ }^{64}$, A. Vásquez-Ramírez ${ }^{30}$, D. Veberič ${ }^{41}$, C. Ventura ${ }^{27}$, I.D. Vergara Quispe ${ }^{4}$, V. Verzi ${ }^{51}$, J. Vicha ${ }^{32}$, J. Vink $^{83}$, S. Vorobiov ${ }^{76}$, H. Wahlberg ${ }^{4}$, C. Watanabe ${ }^{26}$, A.A. Watson ${ }^{c}$, M. Weber ${ }^{40}$, A. Weindl ${ }^{41}$, L. Wiencke ${ }^{86}$, H. Wilczyński ${ }^{70}$, M. Wirtz ${ }^{42}$, D. Wittkowski ${ }^{38}$, B. Wundheiler ${ }^{8}$, A. Yushkov $^{32}$, O. Zapparrata ${ }^{14}$, E. Zas ${ }^{79}$, D. Zavrtanik ${ }^{76,77}$, M. Zavrtanik ${ }^{77,76}$, L. Zehrer ${ }^{76}$

${ }^{1}$ Centro Atómico Bariloche and Instituto Balseiro (CNEA-UNCuyo-CONICET), San Carlos de Bariloche, Argentina

${ }^{2}$ Centro de Investigaciones en Láseres y Aplicaciones, CITEDEF and CONICET, Villa Martelli, Argentina

${ }^{3}$ Departamento de Física and Departamento de Ciencias de la Atmósfera y los Océanos, FCEyN, Universidad de Buenos Aires and CONICET, Buenos Aires, Argentina

${ }^{4}$ IFLP, Universidad Nacional de La Plata and CONICET, La Plata, Argentina

${ }^{5}$ Instituto de Astronomía y Física del Espacio (IAFE, CONICET-UBA), Buenos Aires, Argentina

${ }^{6}$ Instituto de Física de Rosario (IFIR) - CONICET/U.N.R. and Facultad de Ciencias Bioquímicas y Farmacéuticas U.N.R., Rosario, Argentina

${ }^{7}$ Instituto de Tecnologías en Detección y Astropartículas (CNEA, CONICET, UNSAM), and Universidad Tecnológica Nacional - Facultad Regional Mendoza (CONICET/CNEA), Mendoza, Argentina

${ }^{8}$ Instituto de Tecnologías en Detección y Astropartículas (CNEA, CONICET, UNSAM), Buenos Aires, Argentina

${ }^{9}$ International Center of Advanced Studies and Instituto de Ciencias Físicas, ECyT-UNSAM and CONICET, Campus Miguelete - San Martín, Buenos Aires, Argentina

${ }^{10}$ Observatorio Pierre Auger, Malargüe, Argentina

${ }^{11}$ Observatorio Pierre Auger and Comisión Nacional de Energía Atómica, Malargüe, Argentina

12 Universidad Tecnológica Nacional - Facultad Regional Buenos Aires, Buenos Aires, Argentina

${ }^{13}$ University of Adelaide, Adelaide, S.A., Australia

14 Université Libre de Bruxelles (ULB), Brussels, Belgium

15 Vrije Universiteit Brussels, Brussels, Belgium

${ }^{16}$ Centro Brasileiro de Pesquisas Fisicas, Rio de Janeiro, RJ, Brazil

${ }^{17}$ Centro Federal de Educação Tecnológica Celso Suckow da Fonseca, Nova Friburgo, Brazil

${ }^{18}$ Instituto Federal de Educação, Ciência e Tecnologia do Rio de Janeiro (IFRJ), Brazil

${ }^{19}$ Universidade de São Paulo, Escola de Engenharia de Lorena, Lorena, SP, Brazil

${ }^{20}$ Universidade de São Paulo, Instituto de Física de São Carlos, São Carlos, SP, Brazil

${ }^{21}$ Universidade de São Paulo, Instituto de Física, São Paulo, SP, Brazil

${ }^{22}$ Universidade Estadual de Campinas, IFGW, Campinas, SP, Brazil

${ }^{23}$ Universidade Estadual de Feira de Santana, Feira de Santana, Brazil

${ }^{24}$ Universidade Federal do ABC, Santo André, SP, Brazil

${ }^{25}$ Universidade Federal do Paraná, Setor Palotina, Palotina, Brazil

${ }^{26}$ Universidade Federal do Rio de Janeiro, Instituto de Física, Rio de Janeiro, RJ, Brazil

${ }^{27}$ Universidade Federal do Rio de Janeiro (UFRJ), Observatório do Valongo, Rio de Janeiro, RJ, Brazil

${ }^{28}$ Universidade Federal Fluminense, EEIMVR, Volta Redonda, RJ, Brazil

${ }^{29}$ Universidad de Medellín, Medellín, Colombia

${ }^{30}$ Universidad Industrial de Santander, Bucaramanga, Colombia

${ }^{31}$ Charles University, Faculty of Mathematics and Physics, Institute of Particle and Nuclear Physics, Prague, Czech Republic

32 Institute of Physics of the Czech Academy of Sciences, Prague, Czech Republic 
${ }^{33}$ Palacky University, RCPTM, Olomouc, Czech Republic

34 CNRS/IN2P3, IJCLab, Université Paris-Saclay, Orsay, France

${ }^{35}$ Laboratoire de Physique Nucléaire et de Hautes Energies (LPNHE), Sorbonne Université, Université de Paris, CNRSIN2P3, Paris, France

${ }^{36}$ Univ. Grenoble Alpes, CNRS, Grenoble Institute of Engineering Univ. Grenoble Alpes, LPSC-IN2P3, 38000 Grenoble, France

${ }^{37}$ Université Paris-Saclay, CNRS/IN2P3, IJCLab, Orsay, France

38 Bergische Universität Wuppertal, Department of Physics, Wuppertal, Germany

${ }^{39}$ Karlsruhe Institute of Technology (KIT), Institute for Experimental Particle Physics, Karlsruhe, Germany

${ }^{40}$ Karlsruhe Institute of Technology (KIT), Institut für Prozessdatenverarbeitung und Elektronik, Karlsruhe, Germany

${ }^{41}$ Karlsruhe Institute of Technology (KIT), Institute for Astroparticle Physics, Karlsruhe, Germany

${ }^{42}$ RWTH Aachen University, III. Physikalisches Institut A, Aachen, Germany

43 Universität Hamburg, II. Institut für Theoretische Physik, Hamburg, Germany

44 Universität Siegen, Department Physik - Experimentelle Teilchenphysik, Siegen, Germany

${ }^{45}$ Gran Sasso Science Institute, L'Aquila, Italy

46 INFN Laboratori Nazionali del Gran Sasso, Assergi (L'Aquila), Italy

${ }^{47}$ INFN, Sezione di Catania, Catania, Italy

${ }^{48}$ INFN, Sezione di Lecce, Lecce, Italy

${ }^{49}$ INFN, Sezione di Milano, Milano, Italy

${ }^{50}$ INFN, Sezione di Napoli, Napoli, Italy

${ }^{51}$ INFN, Sezione di Roma "Tor Vergata", Roma, Italy

52 INFN, Sezione di Torino, Torino, Italy

53 Istituto di Astrofisica Spaziale e Fisica Cosmica di Palermo (INAF), Palermo, Italy

54 Osservatorio Astrofisico di Torino (INAF), Torino, Italy

55 Politecnico di Milano, Dipartimento di Scienze e Tecnologie Aerospaziali, Milano, Italy

${ }^{56}$ Università del Salento, Dipartimento di Matematica e Fisica "E. De Giorgi”, Lecce, Italy

57 Università dell'Aquila, Dipartimento di Scienze Fisiche e Chimiche, L'Aquila, Italy

58 Università di Catania, Dipartimento di Fisica e Astronomia, Catania, Italy

${ }^{59}$ Università di Milano, Dipartimento di Fisica, Milano, Italy

${ }^{60}$ Università di Napoli "Federico II", Dipartimento di Fisica "Ettore Pancini”, Napoli, Italy

${ }^{61}$ Università di Palermo, Dipartimento di Fisica e Chimica "E. Segrè", Palermo, Italy

62 Università di Roma "Tor Vergata", Dipartimento di Fisica, Roma, Italy

${ }^{63}$ Università Torino, Dipartimento di Fisica, Torino, Italy

${ }^{64}$ Benemérita Universidad Autónoma de Puebla, Puebla, México

${ }^{65}$ Unidad Profesional Interdisciplinaria en Ingeniería y Tecnologías Avanzadas del Instituto Politécnico Nacional (UPIITA-IPN), México, D.F., México

66 Universidad Autónoma de Chiapas, Tuxtla Gutiérrez, Chiapas, México

${ }^{67}$ Universidad Michoacana de San Nicolás de Hidalgo, Morelia, Michoacán, México

${ }^{68}$ Universidad Nacional Autónoma de México, México, D.F., México

${ }^{69}$ Universidad Nacional de San Agustin de Arequipa, Facultad de Ciencias Naturales y Formales, Arequipa, Peru

${ }^{70}$ Institute of Nuclear Physics PAN, Krakow, Poland

${ }^{71}$ University of Łódź, Faculty of High-Energy Astrophysics, Łódź, Poland

${ }^{72}$ Laboratório de Instrumentação e Física Experimental de Partículas - LIP and Instituto Superior Técnico - IST, Universidade de Lisboa - UL, Lisboa, Portugal

73 "Horia Hulubei” National Institute for Physics and Nuclear Engineering, Bucharest-Magurele, Romania

${ }^{74}$ Institute of Space Science, Bucharest-Magurele, Romania

75 University Politehnica of Bucharest, Bucharest, Romania

76 Center for Astrophysics and Cosmology (CAC), University of Nova Gorica, Nova Gorica, Slovenia

${ }^{77}$ Experimental Particle Physics Department, J. Stefan Institute, Ljubljana, Slovenia

78 Universidad de Granada and C.A.F.P.E., Granada, Spain

${ }^{79}$ Instituto Galego de Física de Altas Enerxías (IGFAE), Universidade de Santiago de Compostela, Santiago de Compostela, Spain 
${ }^{80}$ IMAPP, Radboud University Nijmegen, Nijmegen, The Netherlands

${ }^{81}$ Nationaal Instituut voor Kernfysica en Hoge Energie Fysica (NIKHEF), Science Park, Amsterdam, The Netherlands

82 Stichting Astronomisch Onderzoek in Nederland (ASTRON), Dwingeloo, The Netherlands

${ }^{83}$ Universiteit van Amsterdam, Faculty of Science, Amsterdam, The Netherlands

${ }^{84}$ University of Groningen, Kapteyn Astronomical Institute, Groningen, The Netherlands

85 Case Western Reserve University, Cleveland, OH, USA

86 Colorado School of Mines, Golden, CO, USA

${ }^{87}$ Department of Physics and Astronomy, Lehman College, City University of New York, Bronx, NY, USA

${ }^{88}$ Louisiana State University, Baton Rouge, LA, USA

${ }^{89}$ Michigan Technological University, Houghton, MI, USA

${ }^{90}$ New York University, New York, NY, USA

${ }^{91}$ Pennsylvania State University, University Park, PA, USA

92 University of Chicago, Enrico Fermi Institute, Chicago, IL, USA

93 University of Delaware, Department of Physics and Astronomy, Bartol Research Institute, Newark, DE, USA

${ }^{94}$ University of Wisconsin-Madison, Department of Physics and WIPAC, Madison, WI, USA

${ }^{a}$ Fermi National Accelerator Laboratory, Fermilab, Batavia, IL, USA

${ }^{b}$ Max-Planck-Institut für Radioastronomie, Bonn, Germany

${ }^{c}$ School of Physics and Astronomy, University of Leeds, Leeds, United Kingdom

${ }^{d}$ Colorado State University, Fort Collins, CO, USA

$e^{e}$ now at Hakubi Center for Advanced Research and Graduate School of Science, Kyoto University, Kyoto, Japan

$f$ also at University of Bucharest, Physics Department, Bucharest, Romania

\section{Acknowledgments}

Felix Schlüter is supported by the Helmholtz International Research School for Astroparticle Physics and Enabling Technologies (HIRSAP) (grant number HIRS-0009). Simulations for this work were performed on the supercomputer ForHLR II and HOREKA at KIT funded by the Ministry of Science, Research and the Arts Baden-Württemberg and the Federal Ministry of Education and Research. The authors acknowledge support by the state of Baden-Württemberg through bwHPC. 